\title{
Reparação de perfuração ocular em um cão usando pericárdio homólogo preservado em glicerina
}

\author{
Homologous pericardium preserved in glycerin used to repair ocular perforation in a dog \\ Paula Stieven Hünning', Grazziane Maciel Rigon', Wanessa Krüger Beheregaray', \\ Daliana Presser ${ }^{2}$, Marcelo Muccillo 3 \& João Antonio Tadeu Pigatto ${ }^{4}$
}

\begin{abstract}
RESUMO
As lesões de córnea ocorrem frequentemente em cães. Várias técnicas cirúrgicas são propostas para reparar perfuração de córnea, incluindo flapes conjuntivais, ceratoplastias, enxertos corneanos e implantes biológicos. Um caso de perfuração ocular em um cão tratado com pericárdio homólogo conservado em glicerina é apresentado. Oito anos de idade, macho, Pit Bull foi encaminhado ao Serviço de Oftalmologia do Hospital de Clínicas Veterinárias da Universidade Federal do Rio Grande do Sul (UFRGS) com 12 horas de perfuração corneana. O exame oftálmico revelou desconforto ocular, epífora, hiperemia conjuntival e perfuração corneana central com $5 \mathrm{~mm}$ no olho direito. A correção cirúrgica foi realizada utilizando pericárdio homólogo conservado em glicerina. O uso de pericárdio homólogo conservado em glicerina foi efetivo na reparação do defeito corneano, preservando as funções e a anatomia oculares.
\end{abstract}

Descritores: perfuração ocular, pericárdio homólogo, enxerto, canino.

\section{ABSTRACT}

The corneal lesions occur frequently in dogs. Many surgical techniques have been proposed for repair of corneal perforation including conjunctival flaps, keratoplastic procedures, xenografts and biologicals grafts. A case report of ocular perforation in a dog treated sucessfully with homologous pericardium preserved in glycerin is presented. An eight year-old, male, Pit Bull, was presented to the Ophthalmology Service of the Hospital de Clínicas Veterinárias of the Universidade Federal do Rio Grande do Sul (UFRGS), Porto Alegre, RS, Brazil with a 12 hour of a corneal perforation. The ophthalmic examination revealed ocular disconfort, epiphora, conjunctival hyperaemia and a central corneal perforation measuring $5 \mathrm{~mm}$ in the right eye. Surgical repair was performed using homologous pericardium preserved in glycerin. Use of the homologous pericardium preserved in glycerin was effective for repairing corneal defect, preserving oculars function and anatomy.

Keywords: ocular perforation, homologous pericardium, grafts, canine. 


\section{INTRODUÇÃO}

O trauma ocular perfurante é uma importante urgência oftalmológica em cães [12,17,19]. Entre as causas mais comuns de perfuração ocular em cães encontram-se brigas, corpos estranhos, úlceras profundas e descemetoceles [19]. As perfurações oculares podem levar ao comprometimento do bulbo do olho por endoftalmite, glaucoma e atrofia ocular $[11,12,20]$.

O tratamento para as perfurações corneanas é cirúrgico objetivando restaurar a estrutura anatômica e a função do bulbo do olho. As perfurações menores de $3 \mathrm{~mm}$ de diâmetro da córnea podem ser tratadas utilizando sutura e adesivos de cianocrilato. [11,19]. Entretanto, quando são encontradas lesões com maior comprometimento, são necessários procedimentos cirúrgicos objetivando reparar ou substituir a córnea lesada $[10,18,19]$. Vários são os materiais e tecidos utilizados na superfície ocular, incluindo enxertos conjuntivais, membrana biológicas, transposição córneo-escleral, transplante de córnea, entre outros $[2,3,5,6-9,11,12,15,20]$.

Frente a uma situação emergencial e à necessidade de preservar a integridade e a funcionabilidade ocular, o enxerto de membranas biológicas conservadas tem apresentado resultados satisfatórios [2$4,6,8,13]$. Vários métodos podem ser utilizados para a conservação de membranas biológicas, incluindo refrigeração, congelamento, liofilização e glicerina $[14,16]$. Apesar das membranas biológicas terem sido utilizadas com resultados animadores na reparação da superfície ocular em cães, o seu emprego, em casos clínicos ainda é limitado. É relatado o uso de pericárdio homólogo, conservado em glicerina, no tratamento de perfuração ocular em um cão.

\section{RELATO DE CASO}

Um cão de oito anos de idade, macho, da raça Pit Bull foi encaminhado ao Serviço de Oftalmologia Veterinária da Universidade Federal do Rio Grande do Sul (UFRGS), apresentando perfuração ocular por trauma há 12 horas. Ao exame oftálmico, observou-se blefarospasmo, epífora, hiperemia conjuntival, edema de córnea, câmara anterior rasa e uma perfuração central com $5 \mathrm{~mm}$ de diâmetro no bulbo do olho direito (Figura 1).

$\mathrm{O}$ animal foi submetido à anestesia geral para ceratoplastia utilizando enxerto homólogo de pericárdio preservado em glicerina. As condutas operatórias foram conduzidas utilizando microscópio cirúrgico ${ }^{1}$. Realizouse a antissepsia com solução de iodo povidona diluído em solução fisiológica na proporção de 1:50. Posteriormente, foram colocados os panos de campo e fez-se a blefarostase mecânica. Com o auxílio de um trépano de 5,5mm, confeccionou-se um fragmento de pericárdio em formato circular e de dimensão $0,5 \mathrm{~mm}$ maior do que a perfuração da córnea. O pericárdio foi hidratado em solução estéril de cloreto de sódio a $0,9 \%$ e após suturado à córnea utilizando-se fio de poliglactina 910 8- $0^{2}$ e pontos simples separados.

Aplicou-se, pela via subconjuntival $0,1 \mathrm{ml}$ de gentamicina ${ }^{3}$. O pós-operatório imediato incluiu administração de flunixin meglumine ${ }^{4}$, na dose de $1,1 \mathrm{mg} / \mathrm{kg}$, via oral durante 5 dias. Além disso, ampicilina benzatina ${ }^{5}$ na dose de $30 \mathrm{mg} / \mathrm{kg}$, a intervalos de vinte e quatro horas, por via subcutânea, durante 7 dias. Topicamente colírios de ciprofloxacina $0,3 \%{ }^{6}$ e flurbiprofeno ${ }^{7}$, a cada 6 horas, durante 15 dias, e colírio de sulfato de atropina a $1 \% 8$, a cada 12 horas, durante 7 dias. Para evitar autotraumatismo foi indicado o uso de colar elizabetano durante 15 dias.

No pós-operatório imediato, observou-se que a câmara anterior estava refeita sem extravasamento do humor aquoso. A evolução da epitelização foi avaliada utilizando a prova da fluoresceína, a qual foi negativa 15 dias após o procedimento cirúrgico. Aos 30 dias, observou-se intensa neovascularização da córnea, presença de tecido de granulação sobre o local do enxerto e leucoma (Figura 2). Após 45 dias, houve remissão do tecido de granulação e diminuição da neovascularização (Figura 3). Seis meses após procedimento cirúrgico, observou-se câmara anterior profunda, leucoma no local da lesão e transparência do restante da córnea.

O pericárdio homólogo manteve a integridade ocular, restabelecendo a capacidade funcional do bulbo do olho.

\section{DISCUSSÃO}

A perfuração corneana maior de $3 \mathrm{~mm}$ não pode ser suturada ou colada com adesivos de cianocrilato [11,19], logo, no presente caso, optou-se por realizar ceratoplastia com pericárdio homólogo conservado em glicerina.

Vários são os tecidos utilizados para reparar a superfície, incluindo membrana amniótica humana, 


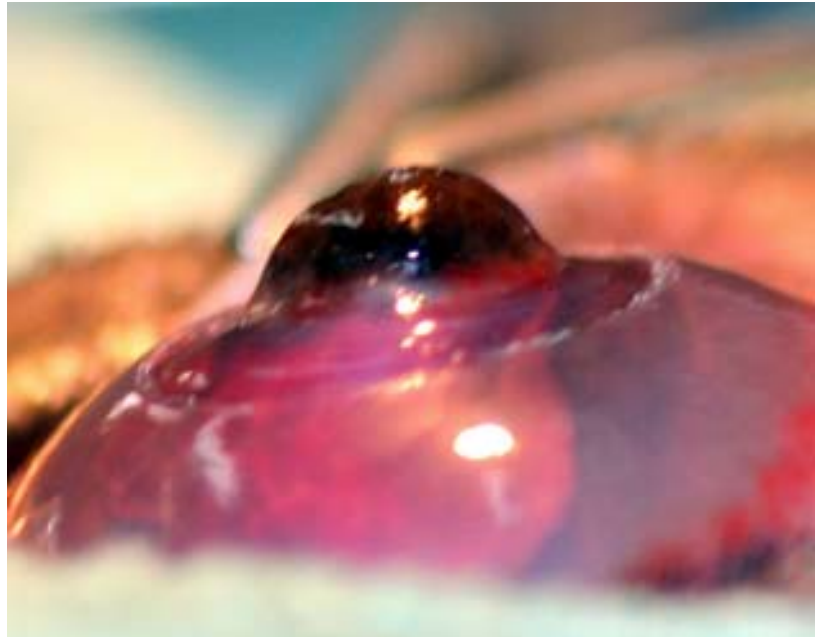

Figura 1. Perfuração do bulbo do olho direito de cão.

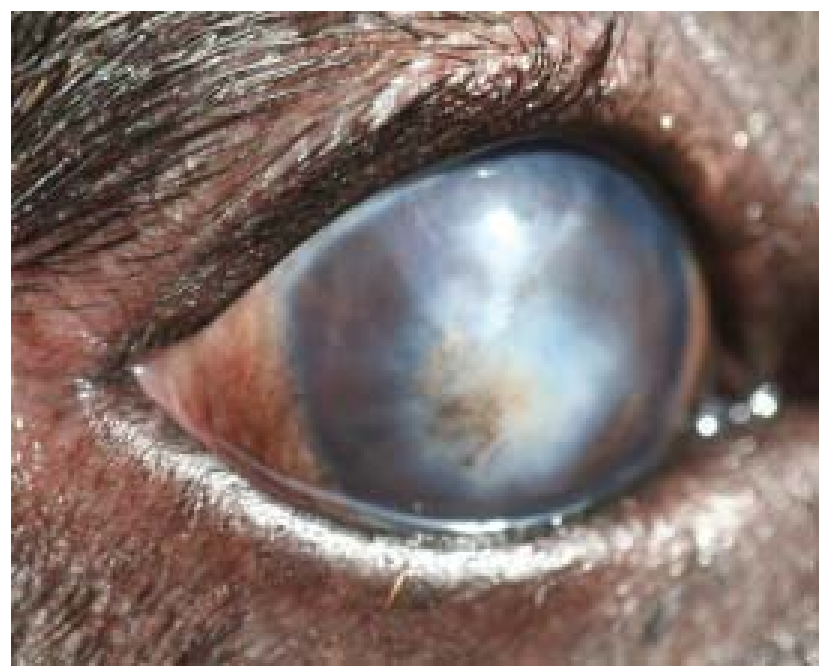

Figura 3. Quarenta e cinco dias após a cirurgia. Evidencia-se leucoma no local da perfuração e transparência do restante da córnea.

equina e lagomorfa, cápsula renal equina, pericárdio equino, submucosa intestinal suína e peritônio canino [1-3,6-9].

A escolha pelo pericárdio deveu-se principalmente aos resultados satisfatórios alcançados em experimentos utilizando membranas biológicas autólogas, homólogas e heteróloga [1,3,6-9,12]. A disponibilidade do tecido e a facilidade de conservação em meio de glicerina motivaram seu uso.

Estudos prévios demonstraram que a glicerina preserva os tecidos mantendo integridade celular e textura, possui ação antibacteriana e diminui a antigenicidade das membranas preservadas [14,16], assim permitindo o uso do pericárdio homólogo como implante. Outras técnicas de conservação de membranas biológicas são preconizadas, tais como refrigeração, congelamento e liofilização $[14,16]$. No entanto,

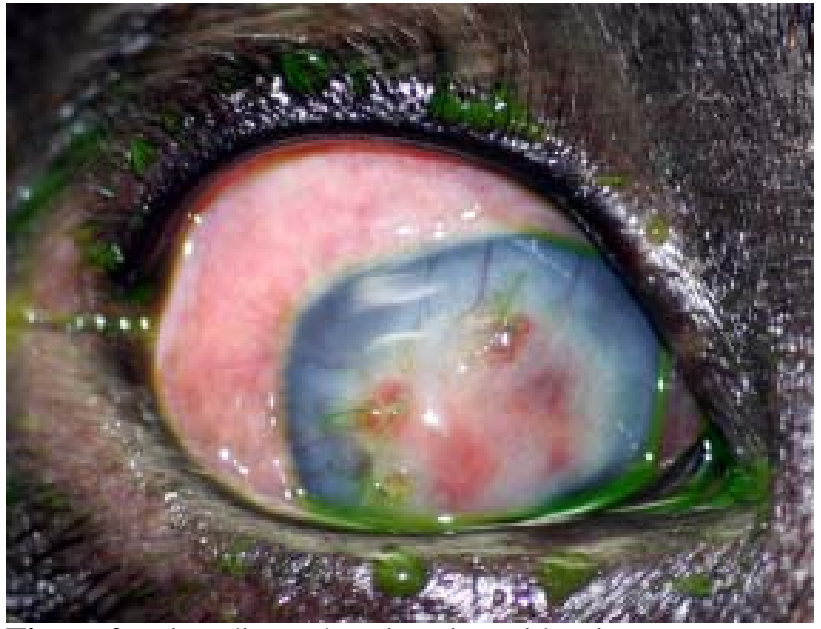

Figura 2. Trinta dias após a cirurgia. Evidenciam-se neovasos e tecido de granulação sobre o local da perfuração. Nota-se hiperemia conjuntival e edema de córnea.

a glicerina foi o método de escolha, por ser simples, barata e de fácil obtenção $[3,6,16]$

O pericárdio foi recortado com dimensão maior que a lesão de córnea, devido à falta de elasticidade para suportar a tensão dos pontos. Também se procurou, com isso, evitar qualquer defeito decorrente da retração do tecido implantado $[8,9]$.

A sutura do enxerto foi realizada com pontos simples separados não penetrantes objetivando reduzir a formação de fibrina e a descompensação da córnea por traumatismo da camada endotelial [15]. Estudos anteriores demonstraram que tanto a utilização de fios de sutura absorvíveis quanto de não absorvíveis apresentaram bons resultados [2,3,6,7,12]. A bibliografia cita que o fio eletivo e indicado para as microcirurgias oftálmicas é o monofilamentar, absorvível ou não, 6-0 a 8-0 [9-12]. No presente caso, a opção pela sutura com pontos simples separados com poliglactina 910 de diâmetro 8-0 mostrou-se adequada a espessura corneana, pois permitiu aposição do enxerto a córnea e não foi observado deiscência de sutura, como foi observado em experimentos com cães e implantação de membrana fetal equina na ceratoplastia lamelar [1,9].

A prova da fluoresceína realizada no período pós-operatório permitiu a avaliação da epitelização sobre o enxerto. Observou-se prova da fluoresceína negativa aos 15 dias de pós-operatório. Comparativamente, o pericárdio equino utilizado na reparação de perfuração de córnea em cães revelou prova da fluoresceína negativa aos 36 dias de pós-operatório [3]. Resultados mais precoces foram verificados com o uso peritônio homólogo canino e membrana 
amniótica equina na ceratectomia lamelar de cães, ocorrendo epitelização da córnea no sétimo e no décimo dias de pós-operatório, respectivamente [2,7].

Estudos prévios demonstraram ausência de rejeição do implante de pericárdio, concluindo tratarse de um bom tecido alternativo para reparos penetrantes da córnea de cães e provavelmente de outras espécies. Também se pode concordar com essa afirmação devido à evolução do processo cicatricial e da resolução do processo inflamatório inicial observado. A presença de leucoma no local do implante corroboram com estudos anteriores $[3,4,6-9,12]$.

O resultado visual das lesões perfurantes dependerá principalmente do diagnóstico precoce, da gravidade da lesão e da eficácia do tratamento. No presente estudo, ocorreu restabelecimento aceitável da transparência no local da lesão e manutenção da integridade anatômica e funcional do bulbo do olho.
Com base nos resultados obtidos, pode-se concluir que o pericárdio homólogo preservado em glicerina mostrou-se viável para o tratamento da perfuração ocular.

\section{NOTAS INFORMATIVAS}

${ }^{1}$ Microscópio cirúrgico MC-M900, DF Vasconcellos S.A., São Paulo, SP

${ }^{2}$ Vicryl ${ }^{\circledR}$, Ethicon, São José dos Campos, São Paulo, SP

${ }^{3}$ Chemitec Agro-Veterinária, Av. Pres. Wilson, 3458, São Paulo-SP

${ }^{4}$ Banamine ${ }^{\circledR}$, Schering Plough, Av. Sir Henry Welcome, 335, Cotia-SP

${ }^{5}$ Optacilin ${ }^{\circledR}$, BYK, Rua do Estilo Barroco, 721, São Paulo-SP ${ }^{6}$ Ciloxan ${ }^{\circledR}$, Alcon, Av. Nossa Sra. da Assunção, 736, São Paulo-SP

${ }^{7}$ Ocufen ${ }^{\circledR}$, Alcon, Av. Nossa Sra. da Assunção, 736, São Paulo-SP

${ }^{8}$ Atropina 1\% ®, Alcon, Av. Nossa Sra. da Assunção, 736, São Paulo-SP

\section{REFERÊNCIAS}

1 Andrade A.L., Laus J.L., Figueiredo F. \& Batista C.M. 1999. The use of preserved equine renal capsule to repair lamellar corneal lesions in normal dogs. Veterinary Ophthalmology. 2: 79-82.

2 Azevedo A.B. 2006. Ceratoplastia lamelar em cães usando membrana amniótica equina: estudo clínico e morfológico. 130f. São Paulo, SP. Tese (Doutorado em Medicina Veterinária) - Programa de Pós-graduação em Cirurgia da Faculdade de Medicina Veterinária e Zootecnia, Universidade de São Paulo.

3 Barros P.S.M., Safatle A.M.V., Malerba T.A. \& Burner Jr. M. 1995. The surgical repair of the cornea of the dog using pericardium as a keratoprosthesis. Brazilian Journal of Veterinary Research and Animal Science. 32: 251-255.

4 Barros P.S.M., Safatle A.M.V. \& Rigueiro M. 1997. Uso de pericárdio de equino conservado em glicerina como enxerto penetrante da córnea de cães. Estudo experimental. Brazilian Journal of Veterinary Research and Animal Science. 34 : 138-141.

5 Brightman A.H., Mclaughlin A.S. \& Brogdon D. 1989. Autogenous lamellar corneal grafting in dogs. Journal of the American Veterinary Medical Association. 195: 469-475.

6 Featherstone H.J., Sansom J. \& Heinrich C.L. 2001. The use of porcine small intestinal submucosa in ten cases of feline corneal diseases. Veterinary Ophthalmology. 4: 147-153.

7 Garcia J.A., Barros P.S.M., Laus J.L., Ferreira A.L. \& Safatle A.M.V. 1996. Implante de peritônio homólogo conservado após ceratectomia lamelar em cães. Brazilian Journal of Veterinary Research and Animal Science. 33: 290-294.

8 Godoy C.A.L. 2005. Estudo comparativo da utilização de membranas amnióticas de coelha e humana como enxerto em ceratoplastia lamelar em coelhos. 94f. São Paulo, SP. Tese (Doutorado em Medicina Veterinária) - Programa de Pósgraduação em Cirurgia da Faculdade de Medicina Veterinária e Zootecnia, Universidade de São Paulo.

9 Godoy C.A.L., Guerra J.L. \& Barros P.S.M. 2002. Ceratoplastia lamelar em cães utilizando membrana fetal equina como enxerto: estudo experimental. Arquivo Brasileiro de Oftalmologia. 65: 545-549.

10 Izaguirre R.L.B., Ibanez G.F.J., Perez O.S., Pereza A.S., Suarez B. \& Lopez H.F.M. 2000. Recubrimientos conjuntivales en perforaciones corneales conjunctival flaps in corneal perforations. Archivos de la Sociedad Española de Oftalmología. 75: 825-829.

11 Kern T.J. 1990. Ulcerative Keratitis. Small Animal Ophthalmology. 20: 643-665.

12 Kuhns E.L. 1979. Conjuntival patch grafts for treatment corneal lesions in dogs. Modern Veterinary Practice. 60: 301-304.

13 Lassaline M.E., Brooks D.E., Franck J.O., Komaromy A.M., Kallberg M.E. \& Gelatt K.N. 2005. Equine amniotic membrane transplantation for corneal ulceration and keratomalacia in three horses. Veterinary Ophthalmology. 8: 311-317.

14 Leite J.B.F., Marques A.F., Gomes O.M. \& Pigossi N. 1979. A glicerina e a preservação de tecidos. Revista Paulista de Medicina. 93: 81-84. 
15 Morales A., Laus J.L., Souza M.S.B., Santos J.M., Figueiredo F. \& Valeri V. 1996. Comparação entre enxertos autógenos livres e pediculados de conjuntiva no reparo de ceratectomias superficias. Estudo experimental no cão (Canis familiarisLinnaeus-1758). Brazilian Journal of Veterinary Research and Animal Science. 33: 28-31.

16 Pigossi N., Raia A., Lex A., Gama A.H., Simanse O., Haddad J., Stolf N.A.G., Zerbini E.J., Miniti A. \& Tenuto R. 1971. Estudo experimental e clínico sobre o emprego, como implante, da dura-máter homógena conservada em glicerina à temperatura ambiente. Revista da Associação Médica Brasileira. 17: 263-278.

17 Scagliotti R.H. 1988. Tarsoconjunctival island graft for the treatment of deep corneal ulcers, descemetoceles, and perforations in 35 dogs and 6 cats. Seminars in Veterinary Medicine and Surgery (Small Animal). 3: 69-76.

18 Schoenau L.S.F., Pippi N.L. \& Schossler J.O.V. 1993. Avaliação clínica preliminar do fechamento comparativo de incisões corneanas com sutura e biofill (película celulósica). Ciência Rural. 23: 173-177.

19 Slatter D. 2005. Fundamentos em oftalmologia veterinária. In: Slatter D. (Ed). Farmacologia ocular e terapêutica. 3.ed. São Paulo: Roca, p.37-74.

20 Startup F.G. 1984. Corneal ulceration in the dog. Journal of Small Animal Practice. 25: 737-752. 